\title{
La experiencia de Aprendizaje en Acción en el barrio de Benicalap: una innovación docente impulsada en el Máster en Cooperación al Desarrollo de la Universitat Politècnica de València
}

\author{
Victoria Pellicer-Sifres ${ }^{\mathrm{a}}$, Alejandra Boni ${ }^{\mathrm{b}}$, Monique Leivas Vargas ${ }^{\mathrm{c}}$, Vania Wassel \\ Antich $^{\text {d }}$ \\ ${ }^{\mathrm{a}}$ INGENIO (CSIC-UPV), Universitat Politècnica de València. vicpelsi@ingenio.upv.es, ${ }^{\mathrm{b}}$ INGENIO \\ (CSIC-UPV), Universitat Politècnica de València. aboni@dpi.upv.es, ${ }^{\mathrm{c}}$ INGENIO (CSIC-UPV), \\ Universitat Politècnica de València. moleivas@ingenio.upv.es, ${ }^{\mathrm{d}}$ Investigadora \\ independiente.vania zz@hotmail.com
}

\begin{abstract}
This article aims to analyze an innovative teaching and learning experience carried out by the "Development Cooperation Master's Degree" taught at the Politechnical University of València (UPV), Spain. During two weeks, the students of the Master carried out an Action Learning process in the neighborhood of Benicalap (Valencia), with the aim to explore how the housing conditions in the neighborhood influence on the youth's learning and aspirations. The process was developed with the participation of three local organization. As outputs of this research, some proposals for improving the neighbourhood were presented to several actors from Benicalap. University exercised then a part of its social responsibility, moving lessons from the classroom to the street. In turn, UPV students acquired social and technical skills for their personal and professional development.
\end{abstract}

Keywords: Action Learning, Innovative pdagogy, Development, Cooperation, Youth'saspirations

\section{Resumen}

Desde el Máster Universitario de Cooperación al Desarrollo de la UPV se impulsó en el barrio de Benicalap una acción de innovación educativa para conectar la universidad con su entorno social. La metodología utilizada fue la del "Aprendizaje en acción”, el cual se define como un proceso de aprendizaje que es aplicable a situaciones donde los y las participantes se enfrentan a problemas complejos y aprenden explorando nuevas oportunidades, en lugar de aplicar las rutinas de sistemas pre-establecidos. Durante 12 días, el alumnado del máster trabajó conjuntamente con tres asociaciones del barrio (el IES Benicalap, con Casa Caridad y con la asociación Ultramarins 154), con el fin de explorar cómo influyen las condiciones de habitabilidad de los y las jóvenes de Benicalap en su aprendizaje. A partir de esta investigación, se lanzaron propuestas de mejora del barrio y se compartieron públicamente con los actores y vecinos/as del barrio. De esta manera la Universidad ejerció en parte su responsabilidad social, trasladando el aula al barrio. A su vez, el alumnado de la UPV adquirió competencias sociales y técnicas que les servirán para su desarrollo personal y profesional. Es esta comunicación, contamos la experiencia, los aprendizajes obtenidos y algunas reflexiones entorno a la metodología de aprendizaje. 
Palabras clave: Aprendizaje en acción, innovación educativa, desarrollo, cooperación, aspiraciones de la juventud

(Nota: el texto de la presente comunicación está basado en la publicación de las mismas autoras: Boni et al, 2017).

\section{Introducción}

La experiencia de Aprendizaje en Acción en el barrio de Benicalap fue una iniciativa educativa impulsada por el Máster en Cooperación al Desarrollo de la UPV, en colaboración con la asociación Ultramarins 154, la ONG Casa Caridad y el Instituto de Educación Secundaria (IES) Benicalap.

Durante los días comprendidos entre el 29 de mayo y el 9 de junio de 2017 los y las estudiantes del Máster en Cooperación de la Universitat de València (UPV) realizaron un proceso de Aprendizaje en Acción en el barrio de Benicalap (Valencia), con el objetivo de explorar cómo influyen las condiciones de habitabilidad del barrio en las aspiraciones de aprendizaje de los y las jóvenes de Benicalap.

\subsection{El Máster en Cooperación al Desarrollo de la UPV y el Aprendizaje en Acción}

El Máster en Cooperación al Desarrollo de la UPV tiene como objetivo formar a profesionales del desarrollo comprometidos, sensibles a los diferentes contextos, con visión crítica y cuya práctica sea a su vez consciente y reflexiva. Por ello, durante la planificación de su itinerario educativo se plantea la integración de metodologías docentes que consigan desarrollar e implementar dichas capacidades.

Con este fin, en el marco de la asignatura de "Procesos de Desarrollo" se consideró apropiado incorporar la metodología de "Aprendizaje en Acción" (en adelante AA). Se considera que este proceso, al ser desarrollado en un contexto con problemáticas reales y a la vez cercanas, contribuye más al desarrollo de dicha práctica crítica y reflexiva que las clases teóricas dentro de las aulas.

En concreto, el AA se define como un proceso de aprendizaje que es aplicable a situaciones donde los y las participantes se enfrentan a problemas complejos y aprenden explorando nuevas oportunidades, en lugar de aplicar las rutinas de sistemas pre-establecidos (Pedler y Burgoyne, 2000). Este tipo de aprendizaje tiene también una dimensión ética (De Carli y Kinnear, 2015), por la cual las experiencias de las participantes forman parte de una amplio tipo de actividades que pueden contribuir positivamente a las iniciativas de las organizaciones locales que acompañan el AA. La contribución puede ser en forma de generar productos (como informes de investigación) o de iniciar procesos (por ej. de diálogo y movilización) que tienen una relevancia para las necesidades y aspiraciones de las organizaciones participantes.

\subsection{Las organizaciones participantes en el proceso de $\mathrm{AA}$ en el barrio de Benicalap}

De lo anterior se desprende que la implicación en el proceso por parte de las organizaciones con las que se desarrolla el AA es un requisito indispensable para poder identificar, 
comprender y analizar las problemáticas así como para poder elaborar propuestas de acción realistas y alineadas con las necesidades de las organizaciones. En nuestra experiencia participaron tres organizaciones del barrio de Benicalap: Ultramarins 154, Casa Caridad y el IES Benicalap.

Ultramarins 154 (UTM154) es una asociación que promueve actividades culturales, lúdicas y gastronómicas en el barrio de Benicalap. Colaboró durante el proceso de AA poniendo a disposición toda su infraestructura para la realización de las diferentes actividades. Durante los 12 días del proceso, fue el lugar de referencia para los encuentros formales e informales, los momentos de trabajo en grupos, las sesiones de formación y los espacios de reflexión en plenario.

Casa Caridad (Asociación Valenciana de Caridad) es una institución sin ánimode lucro, privada, independiente, sin ninguna identidad política o religiosa, cuyo fin es promover la reinserción y prevenir la exclusión social en personas vulnerables. Los y las estudiantes del máster trabajaron concretamente con la Escuela Infantil del centro, que cuenta con 90 niños y niñas en riesgo de exclusión social de entre 1 a 3 años. El objetivo de la escuela, más allá de ofrecer un lugar de convivencia y aprendizaje infantil, es también permitir conciliar la vida laboral y familiar a los padres y madres y facilitarles la búsqueda de empleo.

El IES Benicalap es un centro en el que se imparten diferentes niveles educativos: ESO; bachillerato en las modalidades de Ciencias, Tecnología y Humanidades; ciclos formativos de grado medio (electromecánica y carrocería), de grado superior (automoción, artista fallero y construcción de escenografías) y dos programas de cualificación profesional inicial (carrocería e informática). Los y las estudiantes del máster trabajaron concretamente con dos clases de bachillerato (Humanístico y Mixto) y con una clase de $4^{\circ}$ de la ESO (de Programa de Refuerzo).

Imagen 1: Plano del Barrio Benicalap y ubicación de las organizaciones participantes

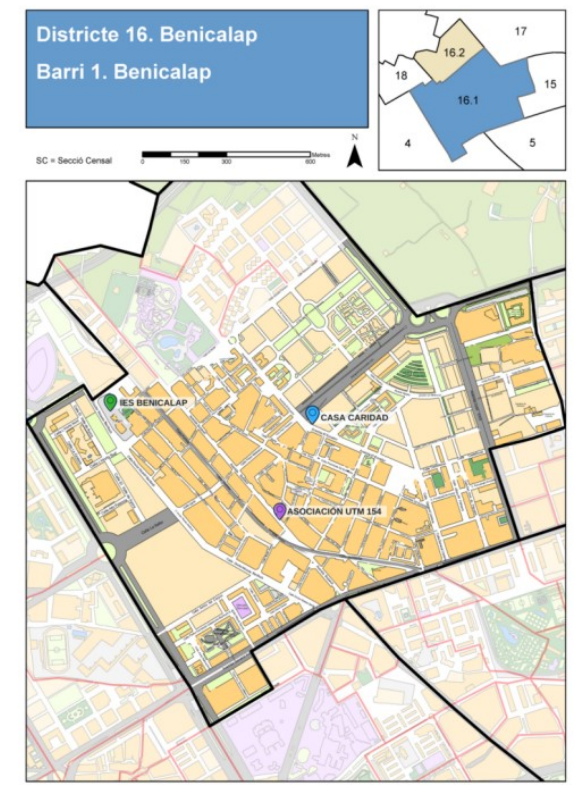

Fuente: Oficina de estadística del Ayuntamiento de Valencia (2016)

En la imagen 1 se muestra la ubicación de las 3 organizaciones en el barrio de Benicalap. Este barrio fue un municipio con autonomía propia hasta finales del siglo XIX, cuando fue 
anexionado a la ciudad de Valencia. Su población censada en 2016 era de 39.635 habitantes (Oficina de Estadística de Valencia, 2016). En los años 50 el barrio estaba habitado por un reducido número de personas, autóctonas, que vivían de trabajar la huerta. Durante los años 60, el barrio de Benicalap crece exponencialmente debido a que recibe inmigración de otros pueblos y ciudades que buscan trabajo en Valencia. Se construyen viviendas de manera no planificada y el barrio se convierte en un barrio dormitorio de la ciudad de Valencia. A partir de los años 70 empiezan las movilizaciones de las vecinas y vecinos del barrio que se agrupan en la Asociación de Benicalap exigiendo demandas básicas como la instalación de alumbrado público, espacios verdes o dotaciones educativas.

En la actualidad, la gran mayoría de la población trabaja en sectores que necesitan poca cualificación. Existe un elevado índice de familias en situación de exclusión social: según el estudio del Ayuntamiento de Valencia sobre áreas vulnerables (2016), el 27,7\% de la población de Benicalap se encuentra en situación de vulnerabilidad. El tejido asociativo es débil y está desvinculado de la juventud del barrio. El patrimonio histórico y cultural de barrio está descuidado por parte de la administración pública y existen muchos solares abandonados.

\section{Objetivos: El proceso de Aprendizaje en Acción en el barrio de Benicalap}

La temática de investigación del proceso de AA fue pensada y dialogada previamente con las organizaciones participantes, con la finalidad de escoger un tema que fuese relevante y de interés para las organizaciones a la vez que viable y realista con las limitaciones del proceso de AA (escaso tiempo y recursos, principalmente).

Se optó por plantear como temática general compartida explorar "cómo las condiciones de habitabilidad de Benicalap influyen en el bienestar de los y las jóvenes del barrio". Entre los estudiantes del máster se formaron cuatro grupos con objetivos de estudio distintos. De esta manera, en función de la naturaleza de cada grupo se concretó y matizó esta pregunta general inicial. (Ver tabla 1).

Tabla 1: Características generales de los grupos

\begin{tabular}{|c|c|c|c|c|}
\hline $\begin{array}{l}\text { Nombre } \\
\text { del grupo }\end{array}$ & $\begin{array}{c}\mathrm{N}^{\mathrm{o}} \\
\text { estudiantes } \\
\text { del máster }\end{array}$ & $\begin{array}{l}\text { Grupo objeto } \\
\text { de estudio }\end{array}$ & ¿Pregunta de investigación? & $\begin{array}{c}\text { ¿Utiliza } \\
\text { herramientas } \\
\text { visuales? }\end{array}$ \\
\hline $\begin{array}{l}\text { Grupo } \\
\text { Proceso } \\
\text { Máster }\end{array}$ & 4 & $\begin{array}{l}\text { El alumnado } \\
\text { del máster ( } 21 \\
\text { estudiantes) }\end{array}$ & $\begin{array}{l}\text { ¿Qué elementos del AA } \\
\text { facilitan u obstaculizan las } \\
\text { aspiraciones de aprendizaje } \\
\text { de los y las estudiantes del } \\
\text { máster? }\end{array}$ & Sí \\
\hline $\begin{array}{l}\text { Grupo } \\
\text { Espacio } \\
\text { Público }\end{array}$ & 4 & $\begin{array}{l}\text { Jóvenes de } \\
\text { Benicalap } \\
\text { (muestra no } \\
\text { predefinida) }\end{array}$ & $\begin{array}{l}\text { ¿De qué manera el espacio } \\
\text { público del barrio de } \\
\text { Benicalap afecta al bienestar } \\
\text { de las/los jóvenes del barrio? }\end{array}$ & Sí \\
\hline $\begin{array}{l}\text { Grupo } \\
\text { Casa } \\
\text { Caridad }\end{array}$ & 7 & $\begin{array}{l}\text { Escuela } \\
\text { Infantil de } \\
\text { Casa Caridad }\end{array}$ & $\begin{array}{l}\text { ¿Cómo las condiciones de } \\
\text { habitabilidad en las que se } \\
\text { encuentran las familias } \\
\text { influyen y afectan al } \\
\text { bienestar y el aprendizaje de }\end{array}$ & No \\
\hline
\end{tabular}




\begin{tabular}{|c|c|c|c|c|}
\hline & & & $\begin{array}{l}\text { los niños y niñas que acuden } \\
\text { a la Escuela Infantil? }\end{array}$ & \\
\hline $\begin{array}{l}\text { Grupo } \\
\text { IES }\end{array}$ & 6 & $\begin{array}{l}3 \text { clases del } \\
\text { IES Benicalap }\end{array}$ & $\begin{array}{l}\text { ¿Cómo influyen las } \\
\text { condiciones de habitabilidad } \\
\text { en las aspiraciones de } \\
\text { aprendizaje de los y las } \\
\text { estudiantes del instituto de } \\
\text { Benicalap? }\end{array}$ & No \\
\hline
\end{tabular}

El primero de los grupos -Grupo Proceso Máster- albergó dos objetivos de naturaleza distinta a los demás: en primer lugar documentar el proceso de AA de los y las estudiantes del máster, y en segundo lugar reflexionar críticamente sobre dicho proceso, analizando qué elementos potenciaron o limitaron el aprendizaje de los y las estudiantes. Fruto del trabajo de este grupo se editó un vídeo resumen del proceso, accesible en http://bit.ly/AA_videoprocesos

El segundo de los grupos -Grupo Espacio Público- exploró cómo las condiciones de habitabilidad del barrio influían en el bienestar de los y las jóvenes. Este grupo se diferenciaba del resto por no contar con un colectivo concreto con quien desarrollar conjuntamente el proceso de AA, de manera que el grupo no disponía a priori de una muestra de estudio acotada. También en este caso, fruto del trabajo del grupo se editó un vídeo resumen con los principales hallazgos, accesible en http://bit.ly/AA_videoespaciopublico

El tercer grupo -Grupo Casa Caridad- trabajó conjuntamente con los padres y madres cuyos hijos/as asisten a la Escuela Infantil. En este caso, cuando se exploraron las condiciones de habitabilidad no únicamente se preguntó por el espacio público del barrio, sino que también se incluyeron cuestiones relativas a la propia Escuela Infantil y a los hogares de las familias. En relación al bienestar, se indagó en referencia al bienestar de los y las niñas que acudían a la escuela.

Finalmente, el cuarto grupo -Grupo Instituto- trabajó conjuntamente con estudiantes de dos clases de $1^{\circ}$ de Bachiller y una clase de $4^{\circ}$ d'E.S.O., así como con profesorado implicado e interesado en el proceso de AA. En este caso, también las condiciones de habitalidad fueron entendidas más allá del espacio público del barrio (se incluyó también condiciones relativas a la infraestructura del propio instituto y a los hogares de los y las alumnas). En cuanto al bienestar, este se concretó acotándose finalmente en torno a la cuestión de los aprendizajes de los estudiantes (aprendizajes obtenidos, potenciados, limitados, aspiraciones, etc.).

Cada grupo contó con el acompañamiento de una facilitadora por parte del equipo de profesorado del máster (que corresponden a las cuatro autoras de esta comunicación).

\section{Desarrollo de la innovación}

El proceso de AA fue planificado en base a cinco fases principales que tuvieron lugar durante los doce días que duró la investigación: 1) Preparación; 2) Diagnóstico; 3 ) Análisis; 4) Propuestas acción y 5) Devolución / difusión de los resultados. En la Figura 1 se representan de manera gráfica. 
Figura 1: Fases del proceso de Aprendizaje en Acción

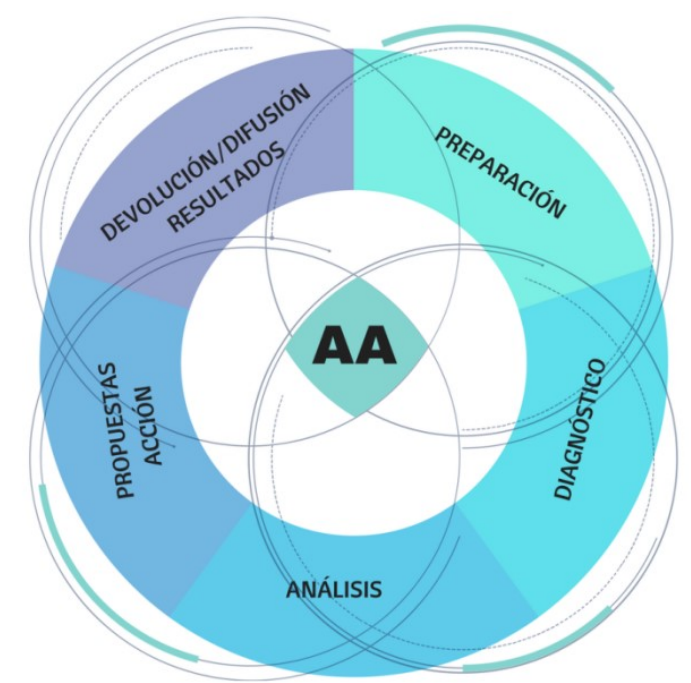

Fuente: Elaboración propia

En cada fase, distintas técnicas de investigación fueron empleadas:

\section{Fase 1: Preparación}

En esta fase (días 1-2), el grupo se familiarizó con el barrio y con los actores con los que cada grupo iba a trabajar. Se realizó un primer recorrido por el barrio para conocer sus lugares de referencia (técnica transecto) y se conocieron a los representantes de las distintas organizaciones. Se presentaron los elementos teóricos que orientarían la investigación y se planificó la agenda de trabajo.

\section{Fase 2: Diagnóstico}

En esta fase (días 2-7) el grupo recabó la información para responder a las preguntas de investigación. Las técnicas empleadas para ellofueron:
* Cuestionarios
* Talleresparticipativos
* Narrativaspersonales
* Entrevistassemi-estructuradas
* Observaciónparticipante

La realidad de cada grupo (su pregunta de investigación, su grupo objeto de estudio, etc) así como la realidad de las organizaciones participantes (su disponibilidad, su capacidad de implicación, etc) supuso que no todos los grupos pudiesen poner en práctica todas las técnicas de investigación. Para mayor detalle sobre las técnicas utilizadas en cada grupo, ver tabla 2.

Tabla 2: Técnicas utilizadas durante la fase de diagnóstico

\begin{tabular}{|c|c|c|c|c|}
\hline Técnica & G1-Proceso & $\begin{array}{l}\text { G2-Espacio } \\
\text { Público }\end{array}$ & $\begin{array}{c}\text { G3-Casas } \\
\text { Caridad }\end{array}$ & G4-Instituto \\
\hline
\end{tabular}

(cc) EY-NC-ND 2018, Universitat Politècnica de València 


\begin{tabular}{|c|c|c|c|c|}
\hline Transecto & $\begin{array}{l}1 \text { transecto en } \\
\text { colectivo, al } \\
\text { inicio del } \\
\text { proceso }\end{array}$ & $\begin{array}{c}\text { Varios } \\
\text { transectos a lo } \\
\text { largo de la } \\
\text { primera semana }\end{array}$ & $\begin{array}{l}1 \text { transecto en } \\
\text { colectivo, al } \\
\text { inicio del } \\
\text { proceso }\end{array}$ & $\begin{array}{l}1 \text { transecto en } \\
\text { colectivo, al } \\
\text { inicio del } \\
\text { proceso }\end{array}$ \\
\hline Cuestionario & $\begin{array}{l}\text { Al inicio y al } \\
\text { final del } \\
\text { proceso }\end{array}$ & $\begin{array}{c}\text { No se realizaron } \\
\text { cuestionario }\end{array}$ & $\begin{array}{c}21 \\
\text { cuestionarios a } \\
\text { los padres y } \\
\text { madres de la } \\
\text { Escuela Infantil }\end{array}$ & $\begin{array}{l}70 \text { cuestionarios } \\
\text { a los y las } \\
\text { estudiantes del } \\
\text { instituto }\end{array}$ \\
\hline $\begin{array}{c}\text { Talleres } \\
\text { Participativos }\end{array}$ & $\begin{array}{l}\text { No se } \\
\text { realizaron } \\
\text { talleres }\end{array}$ & $\begin{array}{c}\text { No se realizaron } \\
\text { talleres }\end{array}$ & $\begin{array}{c}1 \text { taller con } 25 \\
\text { padres y } \\
\text { madres de la } \\
\text { Escuela Infantil }\end{array}$ & $\begin{array}{c}9 \text { sesiones: } 3 \\
\text { talleres en } 3 \\
\text { aulas de } 10-20 \\
\text { estudiantes/aula }\end{array}$ \\
\hline Narrativas & $\begin{array}{l}\text { No se } \\
\text { realizaron } \\
\text { narrativas }\end{array}$ & $\begin{array}{c}\text { No se realizaron } \\
\text { narrativas }\end{array}$ & $\begin{array}{l}\text { No se } \\
\text { realizaron } \\
\text { narrativas }\end{array}$ & $\begin{array}{l}\text { Con } 8 \\
\text { estudiantes del } \\
\text { instituto }\end{array}$ \\
\hline Entrevistas & $\begin{array}{c}\text { Diarias: a los } \\
21 \text { estudiantes } \\
\text { y a las } 4 \\
\text { facilitadoras }\end{array}$ & $\begin{array}{c}\text { A } 12 \text { jóvenes, } 4 \\
\text { adultos y } 2 \\
\text { representantes } \\
\text { de asociaciones } \\
\text { del barrio. Se } \\
\text { utilizaron } \\
\text { fotografías } \\
\text { como recursos }\end{array}$ & $\begin{array}{c}\text { A 3 } \\
\text { trabajadoras } \\
\text { Casa Caridad y } \\
6 \text { familias }\end{array}$ & $\begin{array}{c}\text { A } 4 \\
\text { profesores/as } \\
\text { del instituto }\end{array}$ \\
\hline $\begin{array}{l}\text { Observación } \\
\text { participante }\end{array}$ & $\begin{array}{l}\text { Durante todo el } \\
\text { proceso }\end{array}$ & $\begin{array}{l}\text { Durante todo el } \\
\text { proceso }\end{array}$ & $\begin{array}{c}\text { Durante todo el } \\
\text { proceso }\end{array}$ & $\begin{array}{c}\text { Durante todo el } \\
\text { proceso }\end{array}$ \\
\hline
\end{tabular}

\section{Fase 3: Análisis}

En esta fase (días 6-8) se analizó la información recogida y se obtuvieron los resultados de la investigación. Para ello, se generaron categorías de análisis tomando como referencia los elementos teóricos pertinentes. A lo largo de esta fase se planificaron espacios para compartir con el resto de grupos los hallazgos preliminares y los finales.

\section{Fase 4: Propuestas para la Acción}

Esta fase (días 8-11) se trabajó de manera coordinada con la fase anterior. Los hallazgos preliminares permitieron obtener unas primeras ideas de propuestas para la acción. En el caso de los grupos de Casa Caridad e Instituto, estas propuestas fueron discutidas y complementadas con las organizaciones.

\section{Fase 5: Devolución y difusión de los resultados}

En esta fase (días 11-12) se prepararon los materiales de difusión de los resultados del proyecto: 2 posters explicativos del proceso y 3 posters que sintetizaron los resultados de 
los grupos Espacio Público; Casa Caridad e Instituto, respectivamente. El grupo Espacio Público, además, preparó un vídeo resumen de su experiencia.

El último día del proceso de AA se realizó una presentación pública de los resultados. En la plaza del barrio se expusieron los 5 posters, se presentó el vídeo del grupo Espacio Público y se generó un espacio de diálogo entre los participantes del proceso y los y las vecinas del barrio. Este fue un momento clave para compartir aprendizajes e impresiones, así como para reflexionar sobre cómo el proceso de AA podía estar contribuyendo a mejorar el bienestar de los y las jóvenes de Benicalap

Imagen 2: Estudiantes presentando los pósters durante la presentación pública

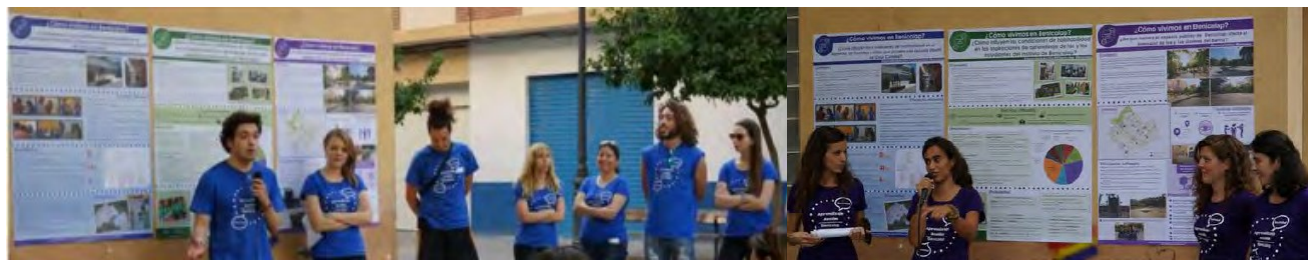

Fuente: Fotografia realizada por Carola Calabuig Tormo

Imagen 3: Estudiantes y vecindario visionando el vídeo durante la presentación pública

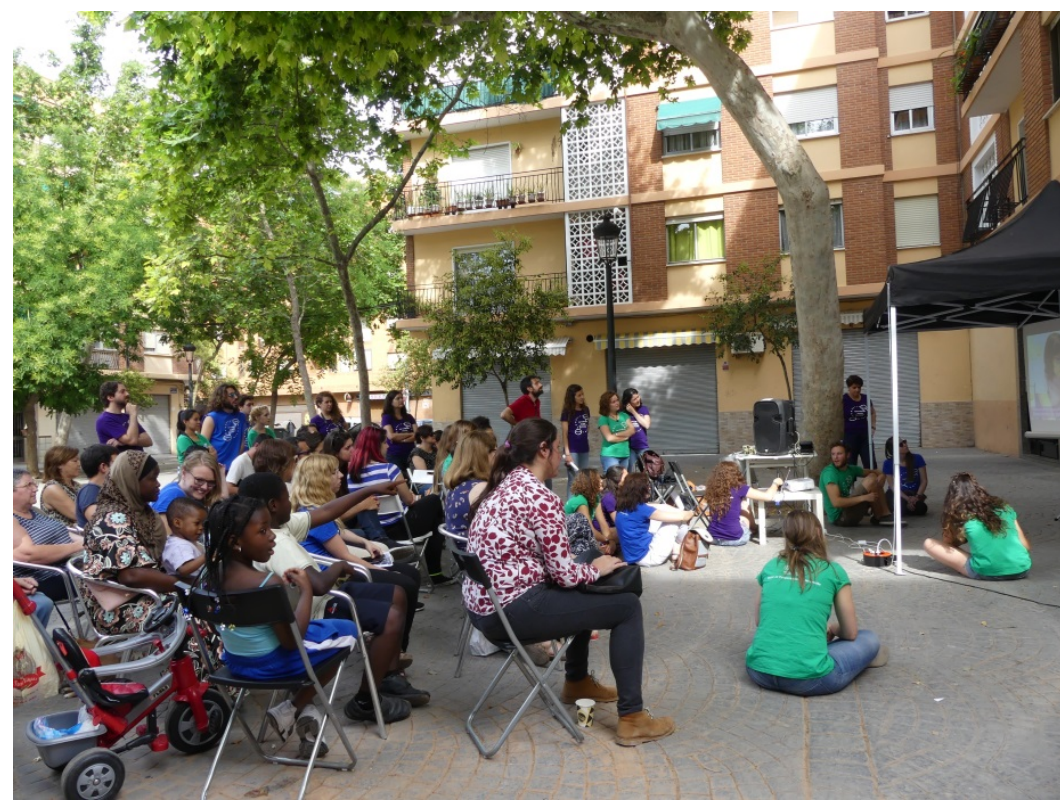

Fuente: Fotografia realizada por Carola Calabuig Tormo

El Grupo 1 - Procesos siguió trabajando durante este día de presentación pública de resultados. En los momentos de reflexión colectiva, recogió evidencias para dar respuesta a su pregunta de investigación y grabó material para ser incluido en el vídeo final del proceso. Este vídeo se presentó a todo el grupo del máster en la sede de Ultramarins, 15 días después de finalizar el AA. Para el grupo de los estudiantes del máster y las facilitadoras del proceso, significó un momento relevante para poder realizar un buen cierre. Habiendo podido tomar perspectiva, asentados algunos aprendizajes, reposadas las emociones y habiendo descansado, los estudiantes recibieron el vídeo producido por las compañeras del Grupo Procesos con gran agradecimiento y con amplio orgullo por los resultados conseguidos.

(cc) EY-NC-ND 2018, Universitat Politècnica de València 


\section{Resultados}

En este apartado planteamos unos resultados generales síntesis de los resultados individuales de cada uno de los cuatro grupos de trabajo.

\subsection{Sobre el espacio público}

Los diferentes grupos con los que se ha trabajado coinciden en afirmar que las dotaciones públicas de las que dispone el vecindario de Benicalap son insuficientes.

Los y las jóvenes demandan más lugares donde poder disfrutar de su ocio de una manera tranquila sin que se les rechace. Aquellos señalan una fuerte presencia policial en las zonas frecuentadas por la juventud, lo que contribuye a incrementar una sensación de malestar entre los y las jóvenes cuando ocupan los espacios públicos. Además, particularmente para las mujeres, la presencia policial no disminuye la sensación de inseguridad.

A las familias usuarias de la Casa de la Caridad también les gustaría que existieran espacios públicos limpios y adaptados para sus hijos e hijas pequeñas. Estos espacios deberían además estar mejor conectados a través del transporte público que también aparece como un limitante para las familias con pocos recursos económicos.

Los y las estudiantes del Instituto también demandan lugares donde reunirse para su ocio pero también para el estudio, y sugieren que el Instituto podría proporcionar algunos de estos espacios si se abriera más al barrio.

En definitiva, parece muy necesaria una mayor inversión pública en dotaciones para el barrio para el ocio y la participación juvenil. Las inversiones hechas en los últimos años (que básicamente se ubican en el nuevo Benicalap) no son accesibles para las personas con menos recursos económicos $\mathrm{y}$ han aumentado la sensación de un barrio dividido $\mathrm{y}$ fragmentado.

\subsection{Condiciones de habitabilidad}

La investigación ha arrojado dos resultados en esta línea; por un lado el estado de las aulas del Instituto no reúne las condiciones para que el aprendizaje se desarrolle adecuadamente. Los y las estudiantes manifiestan que la climatización de las aulas es bastante mejorable, tanto en invierno como en verano.

Con respecto a las familias usuarias de la Casa de la Caridad sí que se percibe que las condiciones de sus viviendas no son las más adecuadas para el bienestar de sus hijos e hijas. Tampoco lo es la limitada oferta de transporte público para poder desplazarse desde sus hogares hasta la Escuela Infantil lo que, en ocasiones, limita el acceso a ese recurso por parte de algunas familias.

En relación con la infraestructura de la Casa de la Caridad, las familias tienen una opinión muy positiva de los espacios que esta institución pone a disposición de sus hijos e hijas, excepto por lo que se refiere a espacios al aire libre de los que no dispone la institución. Esto se ve agravado por la falta de espacios públicos adecuados para los niños y niñas, como ya se ha señalado anteriormente. 
Nuevamente parece necesaria una mayor inversión pública para poder mejorar las condiciones del Instituto, la red de transporte del barrio y la mejora de las condiciones de habitabilidad.

\subsection{Aspiraciones de aprendizaje}

Los y las jóvenes de Benicalap que han participado en la investigación podrían ser considerados como una muestra relativamente representativa de una juventud que vive, crece y camina en un modelo social, marcado por la crisis económica, social y política, y construido en torno a valores capitalistas como el individualismo, el consumo, el dinero como indicador de éxito personal, etc.

Así pues, no es de extrañar que los resultados de los cuestionarios indicasen que la gran mayoría de las personas entrevistadas del Instituto consideraban el aprendizaje como una herramienta meramente instrumental para conseguir un trabajo o tener estabilidad económica; la mayoría de familias entrevistadas de Casa Caridad señalaban la opción "disponer de dinero" como aquello que más puede contribuir a la felicidad de sus hijos/as; y algunos jóvenes del barrio que aceptaron ser entrevistados por el grupo de espacio público explicaron que para ellos el bienestar era "tenerlo todo" o "tener estabilidad económica". No cabe duda de que una situación de precariedad económica tiene consecuencias directas en el bienestar de los y las jóvenes. No obstante, con ello la investigación refleja las consecuencias de un sistema en el que valores como la justicia, el bien común, la participación, la colectividad, las relaciones sociales o la sostenibilidad, por ejemplo, no son las guías de referencia.

Sin embargo, cuando a los y las jóvenes (del Instituto) se les da voz, sí que demandan poner en práctica los canales de participación de los que disponen o reclaman nuevos. Los espacios educativos formales se perfilan como lugares privilegiados en un contexto como el de Benicalap donde fomentar valores distintos a los mayoritarios en la sociedad donde viven los y las jóvenes.

Asimismo, aunque no abordado directamente en la investigación, los espacios asociativos (por ej. grupos de música, danza, cultura, voluntariado, etc.) pueden también ser espacios informales de aprendizaje donde poner en valor la participación, la colectividad, las relaciones sociales, etc. Como se ha señalado anteriormente, Benicalap no cuenta con una oferta de espacios a disposición de los y las jóvenes donde poder reunirse colectivamente y poder tener alternativas. Por ello, un barrio con una oferta de espacios a disposición de las organizaciones y con medidas activas de fomento del asociacionismo tiene mayor potencial para contribuir a la construcción de una juventud más activa $y$ comprometida con valores como la participación colectiva y que le da importancia a las relaciones sociales.

Como consecuencia de lo expuesto anteriormente, las propuestas de los estudiantes del máster de cooperación apuntaban a iniciativas para promover espacios de encuentro y participación entre los jóvenes de Benicalap, bien a través de la reutilización de espacios públicos existentes o con la construcción de espacios nuevos.

\subsection{Redes entre actores}

Tras el proceso de AA realizado y tras el espacio de debate público en la plaza del pueblo el día de la presentación pública, se visibiliza la importancia de conectar organizaciones del Benicalap, públicas y privadas, como son Ultramarins 154, el IES Benicalap, la Casa 
Caridad o la Asociación de Vecinos. El establecimiento de redes entre estas organizaciones sería muy relevante para dos objetivos principalmente:

- Para demandar colectivamente más inversión pública en infraestructura destinada al ocio y a la participación juvenil, la mejora de las condiciones del Instituto así como las condiciones de los parques y jardines del barrio.

- Para generar alianzas sólidas y promover acciones en el barrio que muestren a una juventud implicada y constructiva, al tiempo que reconozcan y potencien, de manera inclusiva y respetuosa, la amplia riqueza cultural del barrio.

El barrio de Benicalap tiene un gran potencial derivado de la gente que lo habita; también tiene un patrimonio muy rico, aunque por las limitaciones temporales de esta investigación no ha podido ser explorado adecuadamente. El esfuerzo conjunto de organizaciones con más trayectoria en el barrio, como la asociación de vecinos, conjuntamente con organizaciones de más reciente creación, como Ultramarins 154, e instituciones como el IES Benicalap o la Casa de la Caridad que están brindando un servicio esencial para el barrio, es necesario para la mejora de las políticas públicas hacia Benicalap y para incrementar las opciones para participar colectivamente y ofrecer otros modelos de convivencia y relaciones sociales. Esto es especialmente relevante para el público juvenil que ha sido el foco principal de nuestra investigación.

\section{Conclusiones}

Finalmente, en este apartado incluimos nuestras conclusiones acerca de la metodología de AA. Como docentes del máster en Cooperación al Desarrollo, sí consideramos positivo y satisfactorio haber incluido esta innovación educativa en el programa docente del máster. Desde nuestro punto de vista, gracias a esta metodología se ha conseguido que el aprendizaje de los y las estudiantes del másterhaya podido ser más profundo y más interiorizado por el hecho de haberse obtenido a través de la propia práctica. Enumeramos a continuación cuatro aspectos clave para los que consideramos que la metodología del AA es una herramienta pedagógica realmente apropiada:

\subsection{EI AA como proceso de aprendizaje relevante para los y las profesionales del desarrollo}

El proceso de AA realizado ha sido valorado muy positivamente entre los y las estudiantes del máster de cooperación. Se realizó un proceso de sistematización y algunas evaluaciones intermedias, y a través de ambas técnicas se comprobó que los y las estudiantes del Máster de Cooperación habían desarrollado habilidades relacionadas con el trabajo en equipo y la facilitación, con la realización de análisis sociales colaborativos o con la traducción y presentación de evidencias de la investigación a audiencias no académicas, entre otras.

El total de los y las estudiantes consideró que el AA había sido una metodología apropiada para poner en prácticas las cuestiones teóricas aprendidas durante el curso, como por ejemplo el uso de las técnicas de investigación, el análisis de datos, la complejidad de los procesos de participación, etc.

\subsection{El AA, un proceso generador de capacidades para el aprendizaje colectivo}


Los cuatro grupos experimentaron, a lo largo de los doce días intensos de trabajo, momentos de tensión, de conflicto, de desencuentros, de necesidad de toma de decisiones rápidas y de frustración. La complejidad de los procesos de desarrollo; el trabajo en tiempo real con organizaciones con agendas, ritmos e intereses distintos; la inseguridad por falta de experiencia; y, en definitiva, la imprevisibilidad de los procesos vivos son factores que forman parte natural del propio proceso de AA. No pueden tenerse en cuenta pero sí puede aprenderse a manejarlos en el momento en el que aparezcan.

A este aspecto hacían referencia los y las estudiantes del máster cuando afirmaban haber puesto en práctica capacidades personales y sociales como la escucha activa, el respeto, la resolución de conflictos y el reconocimiento a las diferencias de opiniones, entre otras. En resumen, podríamos concluir que el proceso de AA ha contribuido a construir y reforzar capacidades con las que superar, de manera colectiva, las barreras encontradas a lo largo del proceso.

\subsection{El AA, un proceso para aprender sobre facilitación}

Un segundo aspecto relevante al que el proceso de AA contribuyó directamente fue a la puesta en práctica del rol de facilitador/a de procesos de desarrollo. A pesar de haber reflexionado durante las clases teóricas acerca de cuán importante son cuestiones como promover una participación real; vigilar y minimizar las dinámicas de poder; cuidar los espacios, los tonos, las palabras; etc., los estudiantes confesaban que fue a través de los talleres y entrevistas realizadas cuando realmente tomaron conciencia de la complejidad y la importancia de todas estas cuestiones.

La gran mayoría de estudiantes marcó el aprendizaje entorno a la figura del dinamizador/a de procesos como uno de los principales retos del proceso, y reconocieron la necesidad de seguir profundizando en este aprendizaje a través de la práctica.

\subsection{El AA, un proceso que reconoce la importancia de los espacios (formales e informales) para compartir y dialogar}

La agenda de los doce días que duró del proceso de AA estaba relativamente organizada, de manera que cada grupo pudiese adaptarla a sus necesidades. Las fases, tal y como se ha explicado en el punto 1.3.2, estaban organizadas según: preparación; diagnóstico; análisis; propuestas acción y devolución de los resultados. No obstante, existieron durante estas fases multitud de espacios de encuentros, de diálogo y de compartir que marcaron la identidad del proceso.

Algunos de estos espacios estaban previamente agendados, como los plenarios para compartir con el resto de grupos la evolución de cada investigación, o la presentación pública en la plaza del pueblo. Otros espacios de intercambio emergieron o sucedieron de manera informal, como los momentos de las comidas, los descansos o los encuentros por el barrio con otros actores. Para los y las estudiantes, tanto unos como otros resultaron fundamentales para compartir emociones, conectar con la realidad de los otros grupos y sentir el proceso de AA como un proceso realmente colectivo. El aprendizaje a este respecto fue el de reconocer la importancia de cuidar, mantener y contribuir a que emerjan estos espacios. 
Como conclusión general, podríamos afirmar que ciertas cuestiones de las aprendidas, útiles y necesarias en la formación de un o una profesional del desarrollo, únicamente pueden llegar a aprenderse a través de una práctica como esta.

\section{Referencias}

- BONI, A., PELLICER-SIFRES, V; LEIVAS M. y WASSEL V. (2017). “¿Cómo vivimos en Benicalap? Una experiencia de Aprendizaje en Acción". En Cuadernos Docentes en Procesos de Desarrollo, vol2.

- DE CARLI, B. y KINNEAR, M. (2015).“Learning amidst urban practices: reflections on Architecture Sans Frontières" en Charrette, vol 2, issue 1, p. 46-56.

- OFICINA D'ESTADÍSTICA DE L'AJUNTAMENT DE VALÈNCIA (2016): Àrees vulnerables a la ciutat de València. Valencia. $<$ http://www.valencia.es/ayuntamiento/webs/estadistica/CatPub/files/Arees\%20Vulnera bles.pdf $>$ [Consulta: 01 de junio de2017]

- PEDLER, M. y BURGOYNE, J.G. (2000). Handbook of Action Research: Participative Inquiry and Practice. London: Sage. 